\title{
Transplante de membrana amniótica associado ao transplante de conjuntiva autólogo no tratamento do pterígio primário
}

\author{
Amniotic membrane transplantation associated with conjunctival autograft for \\ primarypterygium treatment
}

\author{
Alessandra Guerra Daros Castellano ${ }^{1}$ \\ Fernando Antonio Malfatti ${ }^{2}$ \\ Rommel Josué Zagó ${ }^{3}$ \\ Ana Cristina Alvarez Carvalho ${ }^{4}$ \\ Rafael Potrich Reichmann ${ }^{5}$ \\ Hamilton Moreira ${ }^{6}$
}

Trabalho realizado no Serviço de Oftalmologia do Hospital Universitário Evangélico de Curitiba. Curitiba (PR).

Médica Oftalmologista do corpo clínico do Hospital Universitário Evangélico de Curitiba e do Hospital de Olhos do Paraná. Curitiba (PR).

Médico Oftalmologista do Hospital de Olhos do Paraná. Curitiba (PR).

${ }^{3}$ Médico Oftalmologista do corpo clínico do Hospital Universitário Evangélico de Curitiba e do Hospital de Olhos do Paraná. Curitiba (PR).

${ }^{4}$ Residente de Oftalmologia do Hospital Universitário Evangélico de Curitiba. Curitiba (PR).

Residente de Oftalmologia do Hospital Universitário Evangélico de Curitiba. Curitiba (PR).

${ }^{6}$ Doutor em Oftalmologia pela Universidade Federal de São Paulo (UNIFESP). São Paulo (SP); Professor adjunto da Universidade Federal do Paraná. Curitiba (PR) e Professor Assistente da Faculdade Evangélica do Paraná (FEPAR). Curitiba (PR).

Endereço para correspondência: Alessandra Guerra Daros Castellano. Rua Capitão Souza Franco, 833 Apto. 41 - Curitiba (PR) CEP 80730-420

E-mail: alegdcastellano@ hotmail.com

Recebido para publicação em 02.12.2003

Versão revisada recebida em 04.03.2005

Aprovação em 24.05.2005

\begin{tabular}{|l|}
\hline RESUMO \\
\hline Objetivo: Determinar a eficácia do transplante de membrana amniótica \\
associada ao transplante de conjuntiva autólogo no tratamento do pterígio \\
primário. Métodos: Analisou-se prospectivamente 26 pacientes (14 mulhe- \\
res e 12 homens) com idade entre 20 e 60 anos, atendidos no ambulatório \\
de oftalmologia do Hospital Universitário Evangélico de Curitiba e que \\
foram submetidos ao tratamento do pterígio primário com cirurgia de \\
exérese do pterígio e uso de membrana amniótica associada ao transplante \\
de conjuntiva autólogo, entre abril enovembro de 2001. Foram analisados: \\
grau do pterígio, complicações pós-operatórias e recidivas. Resultados: \\
No pós-operatório 4 casos (15,38\%) tiveram complicações, nenhuma \\
considerada séria, sendo a mais freqüente a retração do enxerto em 2 casos. \\
No sexto mês após a cirurgia, 24 pacientes (92,3\%) obtiveram sucesso \\
cirúrgico e 2 pacientes (7,69\%) tiveram recidiva corneana ou conjuntival. \\
Dos pacientes que tiveram complicações, 50\% sofreram recidiva do pterígio \\
(P<0,05). Conclusão: Trata-se de procedimento seguro e com baixo grau \\
de recidiva. As complicações pós-operatórias estão associadas com maior \\
chance de recidiva. É também opção nos casos em que não há conjuntiva \\
doadora suficiente para cobrir toda a área a ser incisada.
\end{tabular}

Descritores: Pterígio/cirurgia; Conjuntiva/transplante; Curativos biológicos; Amnio/transplante; Complicaçõ̃es pós-operatórias; Transplante autólogo; Recidiva

\section{INTRODUÇÃOO}

O pterígio é um processo degenerativo límbico corneano ${ }^{(1)}$ que se apresenta como uma massa fibrovascular, situada em $90 \%$ dos casos nasalmente, podendo invadir a córnea e levar a alterações cosméticas, irritação ocular e déficit visual ${ }^{(2-3)}$. A etiopatogenia continua em processo de investigação, sendo considerados como fatores predisponentes: a exposição ocular aos raios UV, vento, poeira e ainda uma predisposição genética, sugerindo herança auto-sômica dominante ${ }^{(4-5)}$.

O tratamento do pterígio é um tanto controverso, com vários tratamentos sendo indicados na literatura científica ${ }^{(6)}$. São conhecidas as dificuldades técnicas no manejo do pterígio, doença que apresenta um índice de recidiva bastante alta (até $75 \%$ ) com uma média de $30 \%$ que varia conforme o tratamento proposto ${ }^{(3,7)}$. Os índices de recorrência do pterígio primário tratado com o método de excisão simples com exposição escleral são elevados, ocorrendo em até $50 \%{ }^{(1)}$. 
Uma alternativa terapêutica, descrita por vários autores como segura e altamente eficaz, é o transplante de conjuntiva autólogo, que leva a uma menor recidiva se comparado com outras técnicas cirúrgicas, porém o paciente precisa ter conjuntiva bulbar em quantidade suficiente para ser transplanta$\mathrm{da}^{(1,8-10)}$. No entanto em pterígios de cabeça dupla nem sempre se consegue recobrir toda a área de esclera exposta, além disso, deve ser bem avaliada em possíveis candidatos a futuras cirurgias fistulizantes para glaucoma.

A conjuntiva bulbar inferior não é preferida como sítio doador, por ser mais difícil a obtenção de um enxerto largo e maior o risco de desenvolvimento de simbléfaro. Em casos que não é possível ou desejado usar a conjuntiva superior, o uso da inferior é uma opção técnica útil ${ }^{(11-12)}$.

A membrana amniótica consiste de uma camada de epitélio cúbico simples e uma camada basal espessa, composta basicamente de colágeno tipo IV e laminina, e uma matriz estromal avascular, constituindo-se em um bom substrato para o crescimento de células epiteliais, facilitando a epitelização corneana e conjuntival, mantendo o fenótipo epitelial normal ${ }^{(13-14)}$. Ela tem sido empregada em casos onde existe a necessidade de reconstrução da superfície ocular em substituição ao tecido conjuntival como nos casos das doenças cicatriciais corneanas e conjuntivais. O lado estromal da membrana amniótica contém componentes raros da matriz que suprimem a sinalização do TGF- $\beta$ (Fator de crescimento transformador $\beta$ ), proliferacão e diferenciação miofibroblástica de fibroblastos da córnea e do limbo, reduzindo a formação de tecido cicatricial durante a reconstrução da superfície conjuntival e prevenindo a recorrência de tecido cicatricial após a remoção do pterígio ${ }^{(9,15-16)}$.

O trabalho de Prabhasawat et al., que compara o transplante de membrana amniótica com o transplante de conjuntiva autóloga, para o tratamento do pterígio primário, observou que a taxa de recorrência do transplante de conjuntiva foi menor ${ }^{(17)}$.

Este estudo objetiva determinar a eficácia do transplante de membrana amniótica associado ao transplante de conjuntiva autólogo como uma alternativa no tratamento do pterígio primário.

\section{MÉTODOS}

Para este estudo foram selecionados 26 pacientes do Ambulatório de Oftalmologia do Hospital Universitário Evangélico de Curitiba (HUEC), portadores de pterígio primário. A idade mínima para inclusão no protocolo foi de 20 anos e a máxima de 60 anos. Todos foram submetidos a um mesmo protocolo, após concordância, autorização e disponibilidade para o seguimento.

Exame biomicroscópico pré-operatório foi realizado em todos os pacientes para classificá-los em graus, conforme a extensão da invasão corneana do pterígio (Grau I - invasão corneana até $1 \mathrm{~mm}$; Grau II - invasão corneana até $3 \mathrm{~mm}$; Grau III - invasão maior que $4 \mathrm{~mm}$ ).

Após a cirurgia ambulatorial, o paciente recebeu curativo oclusivo e 1 gota de Maxitrol $^{\circledR}$ colírio (neomicina, sulfato de polimixina e dexametasona). Vinte e quatro horas após o curativo oclusivo foi retirado e iniciado a instilação do mesmo colírio de $2 /$ 2 horas, com diminuição gradativa pelo período de 4 semanas. $\mathrm{Na}$ primeira semana foi utilizada lente de contato terapêutica.

Avaliação pós-operatória foi realizada nos dias: $1^{\circ} \stackrel{\circ}{7} 7^{\circ}, 14^{\circ}$, $30^{\circ}$ (retirada de pontos), e nos meses $2^{\circ}, 3^{\circ}$, $4^{\circ}, 5^{\circ}$ e e $6^{\circ}$, visando identificar complicações e observar o tempo médio de ocorrência das recidivas.

O grau de recidiva foi acompanhado segundo a classificação de Abraham Solomon em graus I a IV: Grau I - ausência de vasos episclerais, aparência normal; Grau II - vasos episclerais finos, sem tecido fibrótico na área incisada; Grau III tecido fibrótico na área incisada, mas sem invasão corneana (recidiva conjuntival) e Grau IV - tecido fibrovascular invadindo a córnea (recidiva corneana) $)^{(9,14)}$.

Considerou-se sucesso cirúrgico os olhos que ao final do sexto mês de acompanhamento foram classificados quanto à recidiva nos graus I e II, e insucesso os casos classificados como graus III e IV.

\section{Obtenção e preparo da membrana amniótica humana criopreservada}

- Obtenção da placenta (cesárea eletiva) no Centro Cirúrgico Obstétrico do HUEC, após autorização por escrito e testes sorológicos negativos da parturiente para HIV 1 e 2, Hepatite B e C e sífilis;

- Lavagem da placenta com soro fisiológico 0,9\% em ambiente estéril;

- Separação do âmnion do córion;

- Colocação do âmnion sobre filtro de nitrocelulose estéril com a face epitelial para cima;

- Lavagem do conjunto membrana-filtro com solução tampão-fosfato contendo $1000 \mathrm{U} / \mathrm{ml}$ de penicilina, $20 \mathrm{mcg} / \mathrm{ml}$ de estreptomicina e $2,5 \mathrm{mcg} / \mathrm{ml}$ de anfotericina $\mathrm{B}$;

- Corte do conjunto membrana-filtro em fragmentos de aproximadamente $4 \times 4 \mathrm{~cm} ; \mathrm{e}$

- Colocação de cada fragmento em recipiente estéril contendo glicerol e meio de preservação de córnea na proporção de 1:1 e criopreservação em nitrogênio líquido.

\section{Técnica cirúrgica}

Todos os pacientes foram anestesiados com injeção peribulbar da associação de $3 \mathrm{ml}$ de Xylocaína ${ }^{\circledR} 2 \%$ com $3 \mathrm{ml}$ de Marcaína ${ }^{\circledR} 0,5 \%$.

Realizou-se a ressecção total do pterígio com retirada da cápsula de Tenon subjacente, deixando uma área de esclera exposta. A membrana amniótica foi colocada sobre toda a área da esclera exposta, e sobre a córnea na região onde foi ressecado o pterígio. O lado epitelial para cima e o estromal em contato com a esclera, suturada com pontos episclerais interrompidos de fio Vicry $1^{\circledR} 8.0$.

Na seqüência, realizou-se o transplante de conjuntiva autólogo, retirando-se no sentido fórnice para o limbo, um retalho da conjuntiva bulbar supratemporal, do mesmo olho, com medidas 
de 4x4 mm, com a menor quantidade possível de Tenon aderida a ela. O retalho foi transportado para o local da esclera exposta, respeitando-se a orientação do limbo da conjuntiva transplantada para com a área límbica receptora. A fixação do retalho conjuntival sobre a membrana amniótica foi realizada com pontos interrompidos episclerais de fio nylon 10.0 (Figura 1).

\section{Análise estatística}

Recorreu-se à análise descritiva dos dados através de tabelas. Para a comprovação do objetivo levantado nesse trabalho foram utilizados os testes não-paramétricos "Exato de Fisher" (através do software Epi-Info) e "Coeficiente de Contingência”. O nível de significância (probabilidade de significância) adotado foi menor que $5 \%(\mathrm{p}<0,05)$.

\section{RESULTADOS}

Foram acompanhados 26 olhos de 26 pacientes, sendo 15 homens e 11 mulheres, com uma média de idade de 37,6 anos. Todos os casos eram de pterígio primário e com a cabeça situada nasalmente.

Ao exame biomicroscópico pré-operatório os pterígios foram classificados em 3 graus de invasão sobre a córnea, como mostra a quadro 1. A grande maioria dos casos (16 olhos, $61,53 \%$ ) eram pterígios grau I.

No seguimento pós-operatório tivemos 4 casos $(15,38 \%)$ com complicações, como pode ser visto no quadro 2 , juntamente com o resultado preliminar destes pacientes ao final do seguimento de 6 meses. Não considerada como complicação,

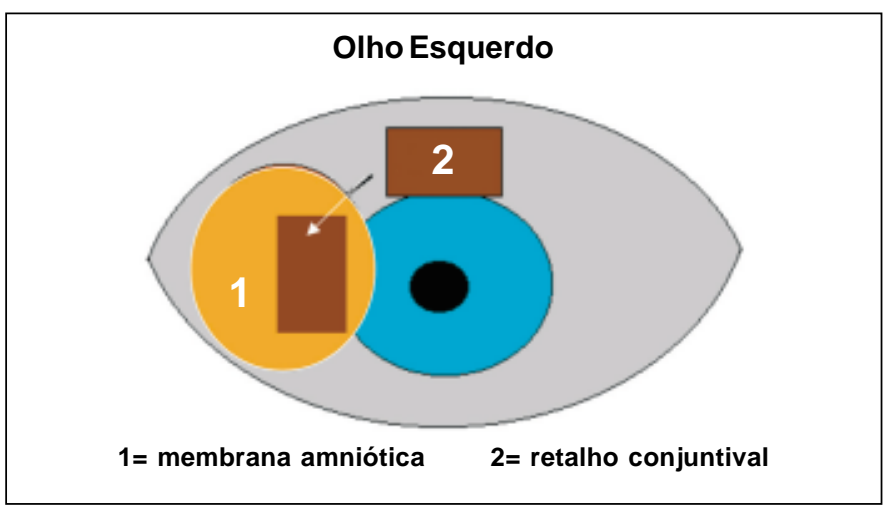

Figura 1 - Modelo esquemático demonstrando a técnica cirúrgica proposta

\begin{tabular}{|lc|}
\hline $\begin{array}{l}\text { Quadro 1.Classificação biomicroscópica pré-operatória do pterígio. } \\
\text { Agrupada em graus de acordo com quantos milímetros o pterígio } \\
\text { invade a superfície corneana }\end{array}$ \\
Grau de & $\begin{array}{c}\text { Número de } \\
\text { pacientes }\end{array}$ \\
invasão corneana & 16 \\
Grau I (até $1 \mathrm{~mm}$ de invasão) & 8 \\
Grau II (até $3 \mathrm{~mm}$ de invasão) & 2 \\
Grau III (4 mm ou mais de invasão)
\end{tabular}

persistiu a hiperemia conjuntival no enxerto ao $3^{\circ}$ mês pósoperatório em $18(69,23 \%)$ dos 26 olhos operados, e ao 6ํ mês em $5(19,23 \%)$ olhos.

A classificação preliminar dos graus de recidiva do estudo proposto encontra-se no quadro 3 . Vinte e quatro pacientes $(92,3 \%)$ foram considerados como sucesso cirúrgico e 2 pacientes $(7,69 \%)$ como recidiva corneana ou conjuntival. O tempo médio da ocorrência das recidivas foi de 4,2 meses.

\section{DISCUSSÃO}

A recorrência após a excisão do pterígio permanece o maior desafio, como evidenciado pela evolução dos numerosos métodos cirúrgicos que foram desenvolvidos nos últimos $\operatorname{anos}^{(16)}$.

A recorrência varia grandemente não apenas com diferentes técnicas cirúrgicas, mas também com diferentes grupos fazendo o mesmo procedimento, e a extensão de conjuntiva comprometida, enfatizando-se a importância da extensa remoção do pterígio ${ }^{(9,17)}$. A grande maioria dos pterígios do nosso estudo se enquadram nos grupos I e II ( 24 dos 26 casos) na classificação biomicroscópica pré-operatória.

Neste estudo, o tempo médio em que ocorreram as recorrências foi de 4,2 meses, semelhante a outros estudos que encontraram 4,8 meses, com somente $6 \%$ das recidivas vistas após 6 meses da cirurgia ${ }^{(18-19)}$.

O grau de recidiva encontrado neste estudo foi de 7,69\% (2 casos), similar a outros autores que tiveram $2 \%$ a $10 \%$ de recidiva do pterígio ${ }^{(17-18,20-21)}$. Os casos que recidivaram eram pacientes que tiveram complicações no pós-operatório, evidenciando uma estreita associação entre complicações (necrose e retração do enxerto de membrana amniótica e conjuntiva) e recidiva do

\begin{tabular}{|l}
$\begin{array}{l}\text { Quadro 2. Complicações pós-operatórias e resultado preliminar no } \\
\text { sexto mês dos pacientes que tiveram complicações }\end{array}$ \\
$\begin{array}{lcc}\text { Numero de } & \text { Resultado } \\
\text { Complicação } & \text { casos } & \text { preliminar } \\
\text { Hematoma no enxerto } & 1 & \text { Grau II } \\
\text { Necrose do enxerto } & 1 & \text { Grau III } \\
\text { Retração do enxerto } & 2 & \text { Graus IV e II } \\
\text { Grau II: vasos episclerais finos na área incisada; Grau III: recidiva conjuntival; } \\
\text { Grau IV: recidiva corneana }\end{array} \begin{array}{l}\text { Obs.: Enxerto representa o conjunto formado pela associação da membrana } \\
\text { amniótica com a conjuntiva autóloga }\end{array}$
\end{tabular}

$\begin{aligned} & \text { Quadro 3. Pacientes agrupados de acordo com o grau de recidiva } \\ & \text { do pterígio no sexto mês de pós-operatório }\end{aligned}$
$\begin{array}{lc}\text { Grau de } & \text { Número de } \\ \text { recidiva do pterígio } & \text { pacientes } \\ \text { Grau I (sucesso) } & 8 \\ \text { Grau II (sucesso) } & 16 \\ \text { Grau III (insucesso) } & 1 \\ \text { Grau IV (insucesso) } & 1 \\ \text { Grau I: aparência normal na área incisada; Grau II: vasos episclerais finos na } \\ \text { área incisada; Grau III: recidiva conjuntival; Grau IV: recidiva corneana }\end{array}$


pterígio $(\mathrm{P}<0,05)$. Nenhuma das complicações encontradas foi considerada séria, e possivelmente estão relacionadas com a má fixação do enxerto na episclera. É possível que a preocupação em não causar perfuração ocular possa ter contribuído para isto. A baixa recorrência, assim como a rara probabilidade de ocorrer uma complicação séria com esta técnica cirúrgica, também foi evidenciada por outros autores ${ }^{(22)}$.

\section{CONCLUSÃO}

Neste estudo observou-se que o transplante de membrana amniótica associado ao transplante de conjuntiva autólogo no tratamento do pterígio primário é um procedimento seguro e com baixo grau de recidiva, semelhante ao do transplante de conjuntiva autólogo. Trata-se de uma boa opção aos casos em que não há conjuntiva doadora suficiente para cobrir toda a área a ser incisada.

Complicações pós-operatórias como retração e necrose do enxerto estão associadas a uma maior chance de recidiva do pterígio.

\section{ABSTRACT}

Purpose: To evaluate the effectiveness of amniotic membrane transplantation associated with conjunctival autograft in primary pterygium treatment. Methods: We have analyzed, prospectively, 26 patients ( 14 women and 12 men), from 20 to 60 years old, examined at the Evangelic Hospital Ophthalmology Outpatient Clinic. They were submitted to primary pterygium treatment with pterygium excision surgery, amniotic membrane transplantation and conjunctival autograft, from April to November, 2001. The evaluated aspects were: degree of pterygium, postoperative complications and recurrence. Results: Four cases $(15,38 \%)$ showed complications, none of them considered serious, conjunctival graft contraction in 2 cases being the most frequent. After a follow-up of 6 months, 24 patients $(92,3 \%)$ had a successful result and 2 patients $(7,69 \%)$ had corneal or conjunctival recurrence. Among the patients with complications 50\% presented pterygium recurrence $(\mathrm{P}<0,05)$. Conclusion: It is a safe surgical procedure with a low degree of recurrence rate. Complications are associated with a greater chance of relapse. This procedure is also an option in cases where there is not sufficient conjunctiva to cover all excised area.

Keywords: Pterygium/surgery; Conjunctiva/transplantation;
Biological dressings; Amnion/transplantation; Postoperative complications; Transplantation, autologous; Recurrence

\section{REFER̂̂NCIAS}

1. Cunha M, Allemann N. Transplante autólogo de conjuntiva no tratamento de pterígio primário e recidivado. Arq Bras Oftalmol. 1993;56(1):78-81.

2. Waller SG, Adamis AP. Pterygium. In: Tasman W, Jaeger EA editors. Duane's clinical ophthalmology. Philadelphia: JB Lipincott; 1994. v. 6, cap 35.

3. Cardillo JÁ, Kara José N, Alves MR, Potério MB, Coelho RP, Ambrósio LE. Instilação do colírio de mitomicina-C no pós-operatório do pterígio primário. Arq Bras Oftalmol. 1995;58(2):138-40.

4. Saw SM, Tan D. Pterygium: prevalence, demography and risk factors. Ophthalmic Epidemiol. 1999;6(3):219-28.

5. Maeda L, Potério MB, Serpa JF, Alves MR, Kara José N. Análise da relação entre ocorrência de pterígio e dominância ocular. Arq Bras Oftalmol. 1996;59 (5):511-3.

6. Hirst LW. The treatment of pterygium. Surv Ophthalmol. 2003;48(2):145-80.

7. Alves MR, Caldeira JAF. Poliose e despigmentação da pele palpebral e regiões periorbitárias após uso local de tiotepa: relato de um caso. Arq Bras Oftalmol. 1991;54(1):27-9.

8. Kenyon KR, Wagoner MD, Hettinger ME. Conjunctival autograft transplantation for advanced and recurrent pterygium. Ophthalmology. 1985;92(11): 1461-70.

9. Prabhasawat P, Barton K, Burkett G, Tseng SC. Comparison of conjunctival autograft, amniotic membrane grafts, and primary closure for pterygium excision. Ophthalmology. 1997;104(6):974-85.

10. Tan D. Conjunctival grafting for ocular surface disease. Curr Opin Ophthalmol. 1999;10(4):277-81.

11. Jap A, Chan C, Lim L, Tan DT. Conjunctival rotation autograft for pterygium. An alternative to conjunctival autografting. Ophthalmology. 1999;106(1):67-71.

12. Syam PP, Eleftheriadis H, Liu CS. Inferior conjunctival autograft for primary pterygia. Ophthalmology. 2003;110(4):806-10.

13. Kim JC, Tseng SC. Transplantation of preserved human amniotic membrane for surface reconstruction in severely damaged rabbit corneas. Córnea. 1995;14(5): 473-84.

14. Shimazaki J, Yang HY, Tsubota K. Amniotic membrane transplantation for ocular surface reconstruction in patients with chemical and thermal burns. Ophthalmology. 1997;104(12):2068-76.

15. Pires RTF, Pires JLTF, Tseng SCG. Transplante de membrana amniótica para reconstrução da superfície corneana e conjuntival. Arq Bras Oftalmol. 1999;62(3): $340-50$.

16. Solomon A, Pires RT, Tseng SC. Amniotic membrane transplantation after extensive removal of primary and recurrent pterygia. Ophthalmology. 2001; 108(3):449-60.

17. Prabhasawat P, Barton K, Burkett G, Tseng SC. Comparison of conjunctival autograft, amniotic membrane grafts, and primary closure for pterygium excision. Ophthalmology. 1997;104(6):974-85.

18. Chen PP, Ariyasu RG, Kaza V, LaBree LD, McDonnell PJ. A randomized trial comparing mitomycin $\mathrm{C}$ and conjunctival autograft after excision of primary pterygium. Am J Ophthalmol. 1995;120(2):151-60.

19. Allan BD, Short P, Crawford GJ, Barret GD, Constable IJ. Pterygium excision with conjunctival auto grafting: an effective and safe technique. $\mathrm{Br} \mathrm{J}$ Ophthalmol. 1993;77(11):698-701.

20. Cohen RA, McDonald MB. Fixation of conjunctival autograft with an organic tissue adhesive. Arch Ophthalmol. 1993;111(9):1167-8.

21. Tananuvat N, Martin T. The results of amniotic membrane transplantation for primary pterygium compared with conjunctival autograft. Cornea. 2004;23(5): 458-63.

22. Chaidaroon W, Wattananikorn S. Conjunctival autograft transplantation for primary pterygium. J Med Assoc Thai. 2003;86(2):111-5. 